01;05.1

\title{
Применение эмпирических потенциалов для расчета упругих свойств графена
}

\author{
(C) А.С. Минкин ${ }^{1}$, И.В. Лебедева ${ }^{2}$, А.М. Попов ${ }^{3}$, А.А. Книжник ${ }^{1,4}$ \\ ${ }^{1}$ Национальный исследовательский центр „Курчатовский институт“, \\ Москва, Россия \\ ${ }^{2}$ Universidad del Pais Vasco UPV/EHU, San Sebastian, Spain \\ ${ }^{3}$ Институт спектроскопии РАН, Москва, Троицк, Россия \\ ${ }^{4}$ ООО „Кинтех Лаб“, Москва, Россия \\ E-mail: amink@mail.ru
}

Поступило в Редакцию 5 февраля 2018 г.

В окончательной редакции 15 ноября 2018 г.

\begin{abstract}
Проведено сравнение упругих свойств плоского слоя графена, рассчитанных с использованием классических эмпирических потенциалов Терсоффа, Бреннера, AIREBO, PPBE-G и LCBOP. Показано, что, несмотря на то что при разработке популярных потенциалов Бреннера и AIREBO формально учитывались упругие характеристики графена, они дают существенные ошибки в значениях модуля Юнга и коэффициента Пуассона. Среди рассмотренных потенциалов наиболее близкие к экспериментальным данным и результатам расчетов из первых принципов значения этих параметров в пределе нулевого удлинения дает потенциал LCBOP. Для количественного моделирования механических явлений в системах на основе графена необходима подгонка параметров потенциала под воспроизведение упругих констант графена с полным учетом деформаций системы и зависимостей этих констант от растяжения.
\end{abstract}

DOI: 10.21883/PJTF.2019.03.47275.17239

Методы классического атомистического моделирования дают уникальную возможность для теоретических исследований явлений в системах, содержащих до $10^{6}$ атомов, на временах до $1 \mathrm{~ms}$. Адекватность такого моделирования определяется выбором потенциала, описывающего межатомное взаимодействие, и подгонкой его параметров под свойства, важные для воспроизведения исследуемых явлений. Способность атомов углерода образовывать различные структуры с двумя, тремя и четырьмя связями между атомами определяет сложность создания адекватного межатомного потенциала для углерода. Были предложены различные потенциалы, параметры которых подогнаны под физические свойства небольших углеводородов и твердые фазы углерода. Наиболее популярные потенциалы, такие как потенциалы Бреннера [1,2] и AIREBO [3], позиционируются как реактивные, т. е. пригодные для описания процессов с разрывом и образованием связей. Однако в ряде задач, исследуемых с помощью атомистического моделирования, разрыва и образования связей не происходит, но важную роль играет точное воспроизведение используемым потенциалом упругих свойств графена. К таким задачам относятся, например, исследование диссипации энергии при относительных колебаниях [4] и движении [5] слоев графена, в том числе в наноэлектромеханических системах [6], исследование структуры дислокаций наложения слоев графена [7] и гетероструктуры графен/нитрид бора [8]. В настоящей работе проводится сравнение упругих констант слоя графена, модуля Юнга и коэффициента Пуассона, рассчитанных с использованием потенциалов Терсоффа [9], Бреннеpa [1,2], AIREBO [3], PPBE-G [10] и LCBOP [11].
Потенциал Терсоффа [9] подгонялся под экспериментальные данные по энергии связи графита, длине связи, энергии связи и объемному модулю упругости алмаза и результаты расчетов из первых принципов энергии связи некоторых других фаз углерода. Этот потенциал послужил основой для разработки серии реактивных потенциалов Бреннера [1,2] и AIREBO [3], которые были подогнаны под экспериментальные данные по длинам связи, энергиям связи и силовым константам небольших углеводородов, графита и алмаза, а также под энергии вакансий в графите и алмазе, рассчитанные методом сильной связи. В AIREBO [3] было добавлено описание межслойного взаимодействия в графите с помощью потенциала Леннарда-Джонса. При подгонке потенциалов [2] и [3] проверялись упругие константы $C_{11}$ и $C_{12}$ графита. LCBOP [11] представляет собой еще одну модификацию потенциала Бреннера с учетом межслойного взаимодействия графита, подогнанную под энергии связи, длины связи и силовые константы графита, алмаза и других фаз углерода по аналогии с потенциалом Терсоффа. В данном случае учитывалась только константа $C_{11}$ графита. Потенциал PPBE-G [10] подгонялся под результаты расчетов из первых принципов межатомных сил для конфигураций графена, полученных в процессе моделирования методом молекулярной динамики.

Степень адекватности описания эмпирическими потенциалами упругих свойств графена можно оценить при сравнении модуля Юнга и коэффициента Пуассона с экспериментальными данными и результатами расчетов из первых принципов. Экспериментальные исследования упругих свойств графита $[12,13]$ показали, что модуль Юнга графита при растяжении вдоль плоскости слоев 
Равновесная длина связи $l$, коэффициент Пуассона $v_{0}$ и модуль Юнга $Y_{0}$ при растяжении в кресельном направлении, рассчитанные различными методами для монослоя графена

\begin{tabular}{l|c|c|c}
\hline \multicolumn{1}{c|}{ Метод } & $l, \AA$ & $v_{0}$ & $Y_{0}, \mathrm{TPa}$ \\
\hline Бреннер, 1990 г. [1] & 1.451 & 0.412 & 0.706 \\
Бреннер, 2002 г. [2] & 1.420 & 0.397 & 0.727 \\
AIREBO [3] & 1.397 & 0.367 & 0.828 \\
Tерсофф [9] & 1.461 & -0.158 & 1.220 \\
PРВE-G [10] & 1.447 & 0.336 & 0.837 \\
LCВОР [11] & 1.420 & 0.221 & 0.943 \\
Эксперимент [12] & & $0.17 \pm 0.02$ & $1.03 \pm 0.02$ \\
Эксперимент [13] & 1.422 & $0.13 \pm 0.03$ & $1.09 \pm 0.02$ \\
Эксперимент [14] & & & $1.0 \pm 0.1$ \\
ТФП [15] & 1.432 & 0.149 & 1.03 \\
ТФП [16] & 1.415 & 0.186 & 1.05 \\
ТФП [17] & 1.430 & 0.174 & 0.99
\end{tabular}

близок к $1 \mathrm{TPa}$ (см. таблицу). Аналогичное значение было измерено для монослоя графена [14] с помощью атомного силового микроскопа в предположении, что эффективная толщина слоя графена равна межслойному расстоянию в графите $h=0.334 \mathrm{~nm}$. Близкие значения модуля Юнга были получены и по теории функционала плотности (ТФП) [15-17]. Экспериментальные измерения $[12,13]$ для графита показали, что коэффициент Пуассона лежит в интервале от 0.10 до 0.19 , а расчеты из первых принципов [15-17] дали значения от 0.15 до 0.19 .

В настоящей работе модуль Юнга и коэффициент Пуассона графена определялись из атомистических расчетов потенциальной энергии при нулевой температуре, в которых проводилась геометрическая оптимизация слоя графена при заданном относительном продольном удлинении $\varepsilon_{x}$. Расчеты с использованием потенциалов Бреннера $[1,2]$ были выполнены с помощью программного пакета MD-kMC [4,5,7], а с использованием потенциалов Терсоффа [9], AIREBO [3], PPBE-G [10] и LCBOP [11] — c помощью программного пакета LAMMPS [18]. Рассматривалась прямоугольная ячейка графена с периодическими граничными условиями. Одна из сторон ячейки растягивалась с шагом $0.01 \%$. Одновременно проводилось сжатие ячейки в поперечном направлении с шагом, который меньше шага для продольного удлинения. Для каждого размера ячейки геометрия слоя оптимизировалась методом сопряженных градиентов до тех пор, пока изменение энергии в последовательных итерациях не составляло менее $10^{-13} \mathrm{eV}$ на атом. Относительное сжатие ячейки $\left(-\varepsilon_{y}\right)$ в поперечном направлении, минимизирующее потенциальную энергию с учетом релаксации структуры, при каждом заданном продольном удлинении $\varepsilon_{x}$ дало зависимость коэффициента Пуассона $v=-\varepsilon_{y} / \varepsilon_{x}$ от продольного удлинения (см. рисунок). В таблице приведен коэффициент Пуассона $v_{0}$, полученный экстраполяцией к нулевому продольному удлинению. Для того чтобы определить модуль Юнга, вторая производная $d^{2} U / d \varepsilon_{x}^{2}$ минимальной потенциальной энергии $U$ на атом графена по отношению к продольному удлинению $\varepsilon_{x}$ рассчитывалась методом конечных разностей. Значение модуля Юнга рассчитывалось экстраполяцией второй производной к нулю: $Y_{0}=\left.\frac{1}{\sigma h} \frac{d^{2} U}{d \varepsilon_{x}^{2}}\right|_{\varepsilon_{x}=0}$ (см. таблицу), где $\sigma=3 \sqrt{3} l^{2} / 4-$ площадь, приходящаяся на один атом в слое графена при равновесной длине связи $l$. Использование для экстраполяции аппроксимаций полиномами первого-четвертого порядка для удлинений до $0.1 \%$ приводит к одинаковым результатам для коэффициента Пуассона и модуля Юнга с точностью до третьего знака после запятой.

Необходимость учета смещений атомов внутри периодической ячейки от их положений в равновесной гексагональной структуре при изменении размера ячейки графена видна из сравнения результатов, полученных с оптимизацией и без нее. Так, для потенциала Бреннера 2002 г. [2] учет релаксации структуры слоя приводит к увеличению коэффициента Пуассона в 3 раза (с 0.139 до 0.397), при этом модуль Юнга, наоборот, уменьшается с 1.038 до $0.727 \mathrm{TPa}$. Аналогично для потенциала Бреннера 1990 г. [1] коэффициент Пуассона увеличивается с 0.158 до 0.412 , а модуль Юнга уменьшается с 1.011 до $0.706 \mathrm{TPa}$ при оптимизации положений атомов. Таким образом, учет релаксации структуры при деформации ячейки моделирования важен для адекватного расчета упругих свойств. Также следует отметить, что размер ячейки моделирования должен хотя бы в 2 раза превышать радиус обрезания потенциала межатомного взаимодействия. При выполнении этого требования значения модуля Юнга и коэффициента Пуассона в наших расчетах менялись при изменении размера ячейки не более чем на $0.001 \%$.

Как видно из таблицы, значения модуля Юнга и коэффициента Пуассона при нулевом удлинении, полученные с помощью разных эмпирических потенциалов, заметно различаются. Хотя все потенциалы предсказывают модуль Юнга для графена порядка $1 \mathrm{TPa}$, наиболее близкие к экспериментальным данным и результатам расчетов из первых принципов значения достигаются с использованием потенциалов PPBE-G [10] и LCBOP [11]. Результаты, полученные с помощью потенциалов Бреннера $[1,2]$ и Терсоффа [9], отличаются от экспериментальных данных и значений, полученных из первых принципов, на 30\% и более. За исключением потенциала Терсоффа, для которого получено отрицательное значение коэффициента Пуассона, остальные потенциалы дают коэффициенты Пуассона в интервале 0.2-0.4. Наиболее близок к экспериментальным результатам и значениям, полученным на основе расчетов из первых принципов, в данном случае LCBOP.

Отметим также различное поведение потенциалов при увеличении растяжения. Так, для потенциала Бреннера 2002 г. [2] коэффициент Пуассона при удлинении до $3 \%$ в кресельном направлении падает в 2 раза (см. рисунок). Аналогично ведет себя потенциал AIREBO [3]. Для сравнения в случае потенциала Бреннера 1990 г. [1] изменение коэффициента Пуассона составляет толь- 


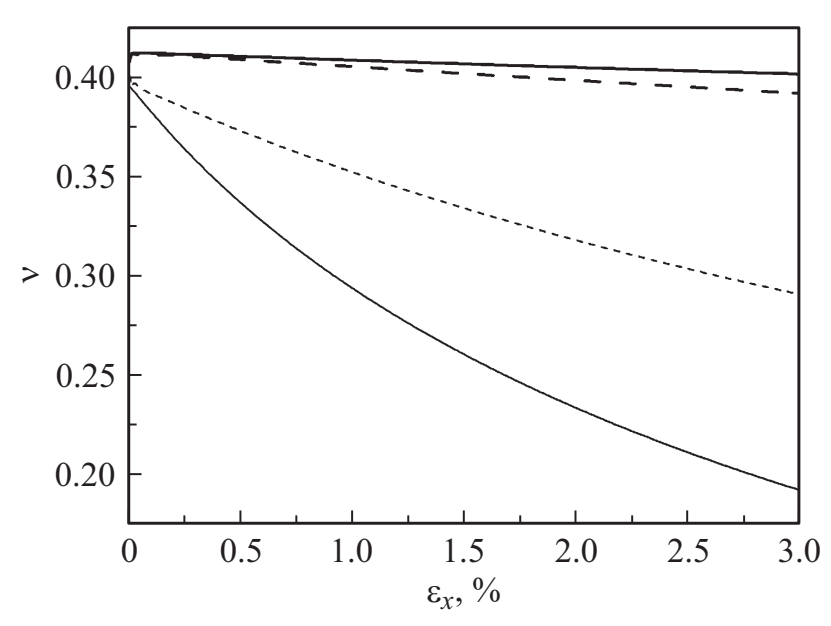

Зависимости коэффициента Пуассона $v$ от продольного удлинения слоя графена $\varepsilon_{x}$, полученные с помощью потенциалов Бреннера 1990 г. [1] (жирные линии) и 2002 г. [2] (тонкие линии) для растяжений вдоль кресельного (сплошные линии) и зигзагообразного (штриховые линии) направлений.

ко 2.6\%. В расчетах из первых принципов [16] при растяжениях 5\% и более коэффициент Пуассона снижался на $3 \%$ на каждый процент удлинения. Кроме того, если в пределе нулевого удлинения коэффициент Пуассона не зависит от направления растяжения, то при конечных удлинениях может наблюдаться заметное различие коэффициентов Пуассона для растяжений в кресельном и зигзагообразном направлениях. Для потенциала Бреннера 2002 г. коэффициент Пуассона при удлинении до 3\% в кресельном направлении оказывается меньше коэффициента Пуассона при аналогичном растяжении в зигзагообразном направлении более чем в 1.5 раза (см. рисунок). Для потенциала Бреннера 1990 г. коэффициент Пуассона несколько ниже при растяжении вдоль зигзагообразного направления. Различие коэффициентов Пуассона при удлинении 3\% в кресельном и зигзагообразном направлениях составляет только $2.4 \%$. Такое поведение ближе к расчетам из первых принципов [16], где коэффициент Пуассона оказался ниже для зигзагообразного направления и различие коэффициентов Пуассона для растяжения в кресельном и зигзагообразном направлениях составляло около 7\% при удлинении $5 \%$.

Таким образом, расчет упругих свойств показывает, что из рассматриваемых потенциалов лучшие результаты в пределе нулевого удлинения достигаются для PPBE-G [10] и LCBOP [11]. Тем не менее в случае PPBE-G коэффициент Пуассона получается заметно выше, а модуль Юнга ниже экспериментальных значений и результатов расчетов на основе ТФП. Кроме того, для этих потенциалов требуются дополнительные исследования зависимостей упругих свойств от растяжения. Хотя формально коэффициент Пуассона и модуль Юнга учтены в подгонке потенциалов Бреннера $[1,2]$ и AIREBO [3], можно предположить, что подгонка проводилась без релаксации структуры при растяжении.
Те же параметры, рассчитанные с учетом такой релаксации, далеки от экспериментальных значений. Поэтому необходим полный учет деформаций структуры при подгонке упругих свойств. Использование нелинейных характеристик, таких как экспериментальные данные и результаты, полученные на основе расчетов из первых принципов, для зависимости коэффициента Пуассона от растяжения, при подгонке эмпирических потенциалов могут позволить дополнительно улучшить их точность.

Работа выполнена при частичной поддержке РФФИ (18-02-00985), Grupos Consolidados UPV/EHU del Gobierno Vasco (IT578-13), EU-H2020 „MOSTOPHOS“ (646259) и с использованием оборудования центра коллективного пользования „Комплекс моделирования и обработки данных исследовательских установок мега-класса“ НИЦ „Курчатовский институт“ (http://ckp.nrcki.ru).

\section{Список литературы}

[1] Brenner D.W. // Phys. Rev. B. 1990. V. 42. P. 9458-9471.

[2] Brenner D.W., Shenderova O.A., Harrison J.A., Stuart S.J., Ni B., Sinnott S.B. // J. Phys.: Condens. Matter. 2002. V. 14. P. 783-802.

[3] Stuart S.J., Tutein A.B., Harrison J.A. // J. Chem. Phys. 2000. V. 112. P. 6472-6486.

[4] Lebedeva I.V., Knizhnik A.A., Popov A.M., Lozovik Y.E., Potapkin B.V. // Phys. Chem. Chem. Phys. 2011. V. 13. P. 5687-5695.

[5] Popov A.M., Lebedeva I.V., Knizhnik A.A., Lozovik Y.E., Potapkin B.V. // Phys. Rev. B. 2011. V. 84. P. 245437 (1-8).

[6] Kang J.W., Lee K.W. // J. Nanosci. Nanotechnol. 2015. V. 15. P. 4895-4899.

[7] Popov A.M., Lebedeva I.V., Knizhnik A.A., Lozovik Y.E., Potapkin B.V. // Phys. Rev. B. 2011. V. 84. P. 045404 (1-6).

[8] Argentero G., Mittelberger A., Monazam M.R.A., Cao Y., Pennycook T.J., Mangler C., Kramberger C., Kotakoski J., Geim A.K., Meyer J.C. // Nano Lett. 2017. V. 17. P. $1409-1416$.

[9] Tersoff J. // Phys. Rev. B. 1988. V. 61. P. 2879-2882.

[10] Wei D., Song Y., Wang F. // J. Chem. Phys. 2011. V. 134. P. 184704 (1-5).

[11] Los L.H., Fasolino A. // Phys. Rev. B. 2003. V. 68. P. 024107 $(1-14)$.

[12] Blakslee O.L., Proctor D.G., Seldin E.J., Spence G.B., Weng T. // J. Appl. Phys. 1970. V. 41. P. 3373-3382.

[13] Bosak A., Krisch M., Mohr M., Maultzsch J., Thomsen C. // Phys. Rev. B. 2007. V. 75. P. 153408 (1-4).

[14] Lee C., Wei X.D., Kysar J.W., Hone J. // Science. 2008. V. 321. P. 385-388.

[15] Kudin K.N., Scuseria G.E., Yakobson B.I. // Phys. Rev. B. 2001. V. 64. P. $235406(1-10)$.

[16] Liu F., Ming P., Li J. // Phys. Rev. B. 2007. V. 76. P. 064120 $(1-7)$.

[17] Lebedeva I.V., Lebedev A.V., Popov A.M., Knizhnik A.A. // Phys. Rev. B. 2016. V. 93. P. 235414 (1-13).

[18] Plimpton S. // J. Comput. Phys. 1995. V. 117. P. 1-42. 